\title{
NEW SIMULATION RESULTS FOR THE ELECTRON-CLOUD EFFECT AT THE PEP-II POSITRON RING*
}

\author{
Y. Cai, S. Heifets and J. Seeman, SLAC, Stanford, CA 94309, USA \\ M. Furman and M. Pivi, LBNL, Berkeley, CA 94720, USA
}

\section{Abstract}

We present simulation results for the emittance blowup due to the head-tail effect induced by the electron-cloud effect (ECE) in the low-energy ring (LER) at the PEP-II B factory at SLAC.

\section{INTRODUCTION}

The ECE has been observed or is expected at many storage rings $[1,2]$. In particular, there is strong evidence for it in the PEP-II positron ring, where it has led to localized vacuum pressure rises and an emittance blowup that has a strong bunch-to-bunch dependence along the bunch train.

In this article we present an attempt to explain the emittance blowup by means of simulations. These simulations are carried out in two separate stages: in the first, we estimate the electron-cloud density for a given fill pattern. In the second, we take the density thus obtained and feed it as an input parameter into a second simulation that computes the beam blowup. The first simulation is done for rigid, nondynamical bunches with a code that has been under development at LBNL for some time $[3,4]$. In the second simulation, the electron cloud is assumed static, and the bunches dynamical. This second part is done with a new code developed at SLAC, described below, using a simple simulation model introduced by Ohmi and Zimmermann [5].

\section{ELECTRON CLOUD}

We consider only the main two sources of electrons, namely: (1) photoelectrons arising from the synchrotron radiation hitting the walls of the vacuum chamber, and (2) secondary emission from electrons hitting the walls. The model used for the secondary emission yield (SEY) of the TiN-coated chamber is described elsewhere $[3,4]$; we have slightly updated it, however, by fitting its parameters to newer measurements obtained at SLAC, both of the SEY and the emitted-electron energy spectrum [6], of TiN-coated aluminum samples of the PEP-II LER vacuum chamber.

We assume a fill pattern whose basic bunch spacing is 4 RF buckets, and has 8 gaps along the circumference, as shown in Fig. 1. Figure 2 shows the simulated density of the electron cloud for the case of $N_{b}=5 \times 10^{10}$ positrons per bunch, assuming an effective quantum efficiency per

\footnotetext{
* Work supported by the US DOE under contracts no. DE-AC0376 SF00515 and DE-AC03-76SF00098, and by the SNS project. To be published in the Proc. PAC01, Chicago, June 18-22, 2001.
}

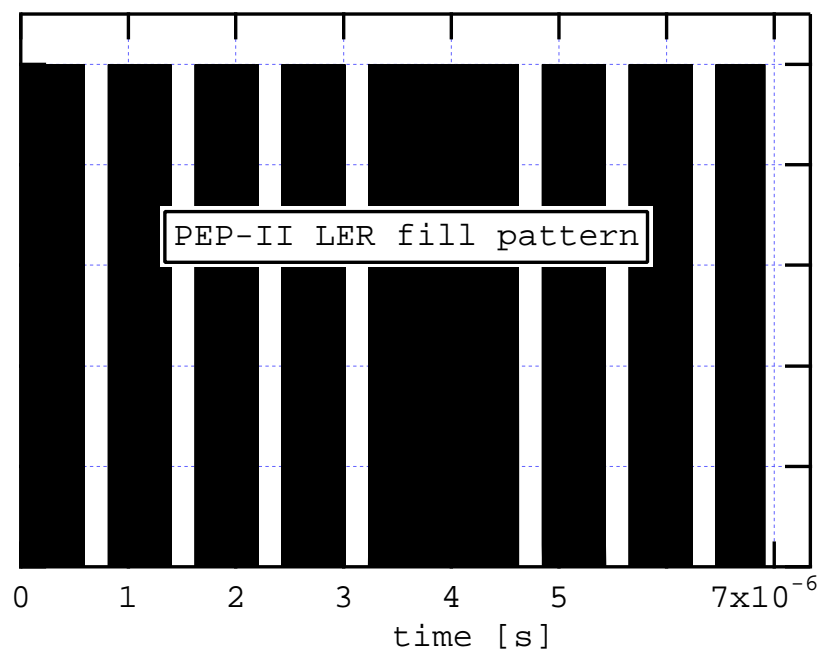

Figure 1: Fill pattern used for the simulation (one full revolution).

penetrated photon $Y^{\prime}=1$. Here we only count those electrons within $5 \mathrm{~mm}$ of the beam axis, since it is only these that contribute significantly to the emittance blowup.

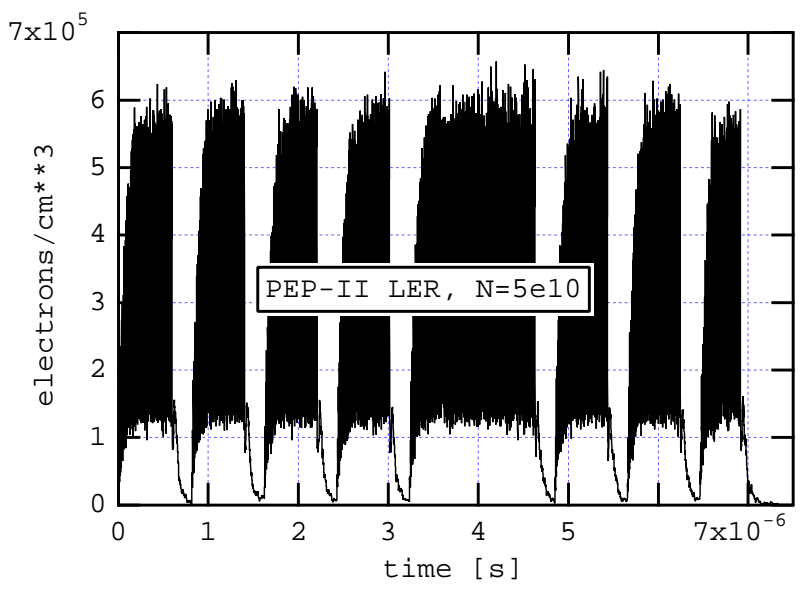

Figure 2: Density of the electron cloud along the bunch train inside a circle of radius $5 \mathrm{~mm}$ about the center of the chamber. 


\section{EMITTANCE BLOWUP}

An object-oriented C++ class library has been written to simulate the interaction between the electron cloud and positron beam. In the library, the electron cloud and positron beam are independent objects that can be constructed by the user. There is no limitation on how many objects of cloud are allowed in the simulation, and the clouds can have different parameters as instances of the cloud class. These features provide us with great flexibility to study various phenomena of the ECE.

We represent the transverse distribution of the electron cloud by $N_{m}$ macroparticles at a given location $s$ in the ring,

$$
f_{\perp}^{-}\left(\vec{x}_{e}, \vec{v}_{e} ; s\right)=\frac{1}{N_{m}} \sum_{n=1}^{N_{m}} \delta\left(\vec{x}_{e}-\overrightarrow{x_{e n}}(s)\right) \delta\left(\vec{v}_{e}-\vec{v}_{e n}(s)\right),
$$

where $\vec{x}_{e}$ and $\vec{v}_{e}$ represent the transverse coordinate and velocity of the electrons. The distribution of the positron bunch is represented by $N_{s}$ longitudinal macro slices. All slices are assumed to have a rigid Gaussian distribution of transverse rms sizes $\left(\sigma_{x}, \sigma_{y}\right)$. The centroid of each slice is treated as a dynamical variable in 6D phase space.

Transversely, we use the 2D vectors $\vec{x}_{c}$ and $\vec{p}_{c}$ to describe the centroid coordinate and canonical momentum of the slices. At the beginning of the simulation, all the transverse coordinates and momenta of the slice centroids are set to zero. Longitudinally, the centroid coordinate $z$ and momentum $p_{z}$ of the slices are initialized to a Gaussian distribution with rms bunch length $\sigma_{z}$ and energy spread $\sigma_{p}$, respectively.

To speed up the simulation, all electrons are lumped into one single slice at a given azimuthal location $s$ with average $\beta$ function. This approximation is justified because we know that the head-tail instability is rather insensitive to the location of the impedance. Before the arrival of the positron bunch, the distribution of electron cloud is reinitialized to a Gaussian distribution with sizes $\sigma_{x}^{c}$ and $\sigma_{y}^{c}$ and the velocities of the electrons are reset to zero. The slices of the bunch are sorted according to their longitudinal position. Starting with the head, the slices collide with the electron cloud sequentially in time. Under the assumption of Gaussian distribution, the kick experienced by $i^{t h}$ electron from the electric field of $n^{\text {th }}$ slice is

$$
\delta \vec{v}_{e i}=-\frac{2 N_{b} r_{e} c}{N_{s}} \overrightarrow{F_{G}}\left(\overrightarrow{x_{e i}}-\overrightarrow{x_{c n}} ; \vec{\sigma}\right)
$$

where $\overrightarrow{F_{G}}$ is given by the Erskine-Bassetti formula [7]. The kick by the electron cloud to the centroid of the slice is expressed as

$$
\delta \vec{p}_{c n}=-\frac{2 r_{e} N_{e}}{N_{m} \gamma} \sum_{i=1}^{N_{m}} \overrightarrow{F_{G}}\left(\overrightarrow{x_{c n}}-\overrightarrow{x_{e i}} ; \vec{\sigma}\right)
$$

where $N_{e}=2 \pi \sigma_{x}^{c} \sigma_{y}^{c} C n_{e}$. Note that the distribution of the electron cloud is not directly used in the calculation and the expression is based on the conservation of the momentum. Because the transverse size of the electron cloud is much larger than that of the beam, the approximation is adequate. Between the collision of two adjacent slices the electrons drift, $\delta \overrightarrow{x_{e}}=\overrightarrow{v_{e}} d z / c$, where $d z$ is the slice separation.

To see the dynamical effects of the positron beam, we track the centroid of the slices with betatron and synchrotron motions. The transverse coordinates are tracked by applying a simple linear Courant-Snyder map around the ring with tunes $\nu_{x}$ and $\nu_{y}$ and lattice functions $\beta_{x}, \alpha_{x}$, etc. In the longitudinal plane similar formulas are applied, with $\beta_{z}=\sigma_{z} / \sigma_{p}$ and $\alpha_{z}=0$.

Current operating parameters of the LER are listed in Table 1. All wiggler magnets in the machine are turned off for higher luminosity. The vertical emittance is estimated from the beam-beam luminosity scan. The average of the beta function is computed using the values at the end of every element. The bunch charge $N_{b}$ is chosen to correspond to the highest operating current in the ring.

Table 1: Parameters for the LER.

\begin{tabular}{llr}
\hline \hline Parameter & Description & Value \\
\hline$E[\mathrm{Gev}]$ & beam energy & 3.1 \\
$\beta_{x}[\mathrm{~m}]$ & average x beta & 9.370 \\
$\beta_{y}[\mathrm{~m}]$ & average y beta & 12.47 \\
$\epsilon_{x}[\mathrm{~nm}-\mathrm{rad}]$ & x emittance & 24.0 \\
$\epsilon_{y}[\mathrm{~nm}-\mathrm{rad}]$ & y emittance & 1.50 \\
$\sigma_{z}[\mathrm{~cm}]$ & rms bunch length & 1.30 \\
$\sigma_{p}$ & relative energy spread & $7.7 \times 10^{-4}$ \\
$\nu_{x}$ & x tune & 0.649 \\
$\nu_{y}$ & y tune & 0.564 \\
$\nu_{s}$ & synchrotron tune & 0.0251 \\
$C[\mathrm{~m}]$ & circumference & 2200 \\
$N_{b}$ & positrons/bunch & $1 \times 10^{11}$ \\
$\alpha$ & momentum compaction & $1.23 \times 10^{-3}$ \\
\hline \hline
\end{tabular}

The other parameters used in the simulations are listed in Table 2. The parameters related to the electron cloud are not yet well established. In Fig. 2 we see that the saturation density is $n_{e} \simeq 6 \times 10^{5} \mathrm{~cm}^{-3}$ for $N_{b}=5 \times 10^{10}$. Other simulations show that $n_{e}$ is higher at higher $N_{b}$, but we do not yet have a reliable estimate. The number of slices in the bunch and macro particles for the electron cloud is balanced between a better accuracy and computational time. They are limited by the requirement of 24 hours turnaround time on a computer workstation. These parameters are always used in the simulation unless statements to the contrary in the text.

\section{RESULTS AND DISCUSSION}

Based on the analytical derivation of the wake field generated by the electron cloud and the theory of instability in the previous section, it is clear that there is a threshold of strong head-tail instability $\left(\xi_{x}=\xi_{y}=0\right)$. In this section 
Table 2: Simulation parameters.

\begin{tabular}{llr}
\hline \hline Parameter & Description & Value \\
\hline$n_{e}\left[\mathrm{~cm}^{-3}\right]$ & e-cloud density & $8.0 \times 10^{5}$ \\
$\sigma_{x}^{c}[\mathrm{~mm}]$ & e-cloud x size & 6.0 \\
$\sigma_{y}^{c}[\mathrm{~mm}]$ & e-cloud y size & 3.0 \\
$N_{s}$ & no. of slices & 1024 \\
$N_{m}$ & no. of macro particles & 10240 \\
\hline \hline
\end{tabular}

we simulate the interaction between the electron cloud and the positron beam and the beam dynamics to compare the result with the analytical approximation. We vary the intensity of the electron cloud $n_{e}$ from $1 \times 10^{5}$ to $1 \times 10^{6} \mathrm{~cm}^{-3}$ and find that the vertical instability approximately occurs at $5 \times 10^{5} \mathrm{~cm}^{-3}$ as shown in Fig. 3. The turn-by-turn evolution of the rms value of slices is plotted in the figure. One can see that, beyond the threshold, the centroids of the slices spread widely comparing to the beam size, leading to an effective increase of the emittance of the beam. To quantify the emittance growth, we define $\Sigma_{y}=\left(\sigma_{y}^{2}+\sigma_{c, y}^{2}\right)^{1 / 2}$, where $\sigma_{c, y}$ is the vertical rms variation of the centroid, and plot it as a function of the cloud density in the second plot. $\Sigma_{y}$ can be measured with a synchrotron light monitor.
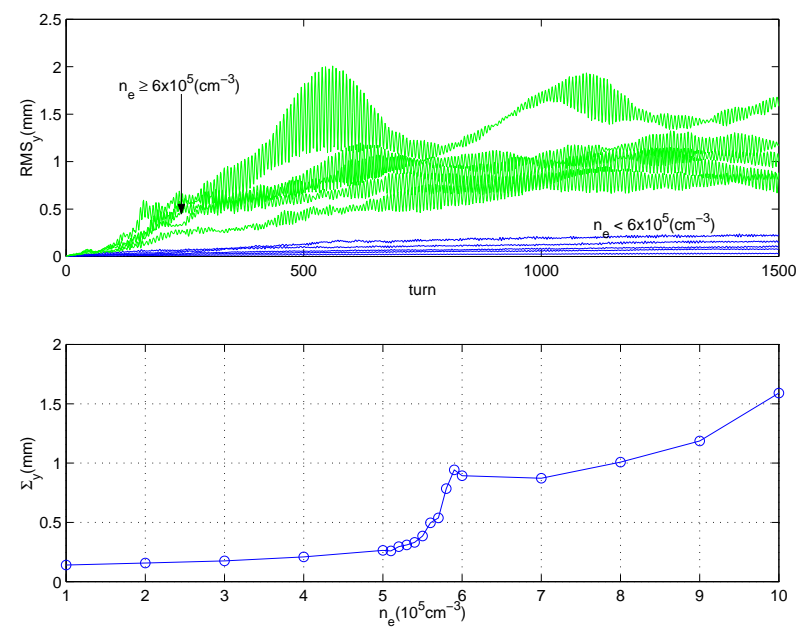

Figure 3: Threshold of head-tail instability caused by electron cloud.

It is well known that the lattice chromaticities can play an important role to stabilize the conventional head-tail instability. To study this effect, we vary $\xi_{y}$ in the range $-10<\xi_{y}<10$ while fixing $\xi_{x}=0$ and $n_{e}=8 \times 10^{5}$ $\mathrm{cm}^{-3}$, which is above the instability threshold. The results are shown in Fig. 4. One can see that $\xi_{y}<0$ leads to large beam blowup while $\xi_{x}>0$ does not, in agreement with experimental observations.

In conclusion, we see clear threshold behaviors of beam blowup as a function of electron cloud density and chromaticity. The actual value of the electron cloud density remains to be better determined. A clear signal of bunch- to-bunch variation of the electron density along the train, if it exists, will require further simulations with much better statistics.
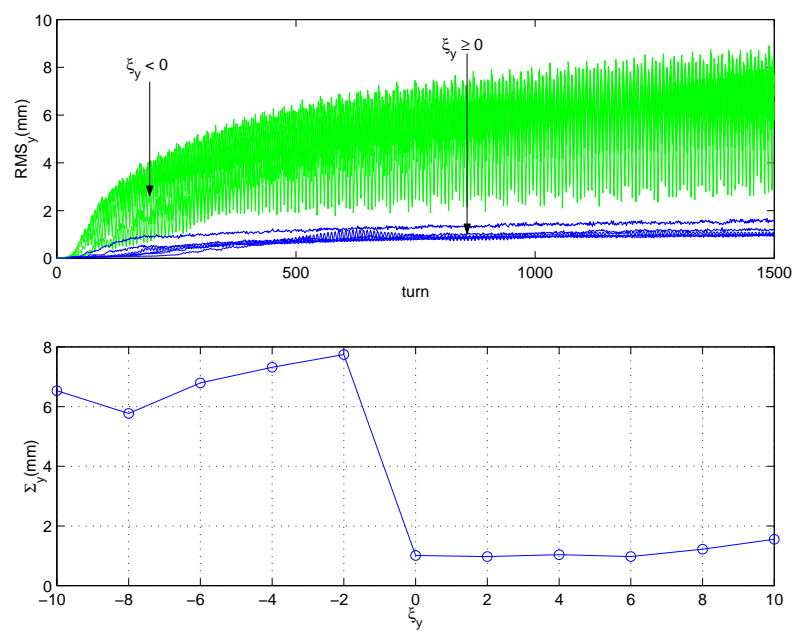

Figure 4: Effective beam size as a function of chromaticity.

\section{ACKNOWLEDGMENTS}

We are grateful to R. Kirby for discussions on the SEY, and to NERSC for supercomputer support.

\section{REFERENCES}

[1] For a summary, see Proc. ICFA Workshop on TwoStream Instabilities, Santa Fe, NM, Feb. 16-18, 2000, http://www.aps.anl.gov/conferences/icfa/two-stream.html

[2] G. Rumolo, F. Ruggiero and F. Zimmermann, PRST-AB 4, 012801 (2001) (erratum: 4, 029901 (2001)). See also F. Zimmermann, these proceedings.

[3] M. A. Furman and G. R. Lambertson, Proc. Intl. Workshop on Multibunch Instabilities in Future Electron and Positron Accelerators (MBI-97), KEK, Tsukuba, Japan, 1518 July 1997 (KEK Proceedings 97-17, Dec. 1997, p. 170); http://www.lbl.gov/ miguel/MBI97-ECI-PEPII.pdf

[4] M. A. Furman, LBNL-41482/CBP Note 247/LHC Project Report 180, May 20, 1998, http://www.lbl.gov/ miguel/ LHCpr180.pdf

[5] K. Ohmi and F. Zimmermann, Phys. Rev. Lett. 853821 (2000).

[6] R. E. Kirby and F. K. King, SLAC-PUB-8212, Oct. 2000, to be published in NIMPR.

[7] M. Bassetti and G. Erskine, CERN ISR TH/80-06 (1980).

[8] H. Fukuma et al., Proc. EPAC2000 (Vienna), p. 1124. 